http://www.jfas.info

\title{
RECOGNITION OF HUMAN GAIT IN OBLIQUE AND FRONTAL VIEWS USING KINECT
}

\author{
R. Sahak, I. Yassin, N. M. Tahir ${ }^{*}$, F. H. K. Zaman and A. Zabidi \\ Faculty of Electrical Engineering, Universiti Teknologi MARA, 40450 Shah Alam, Selangor, \\ Malaysia
}

Published online: 05 October 2017

\begin{abstract}
This study describes the recognition of human gait in the oblique and frontal views using novel gait features derived from the skeleton joints provided by Kinect. In D-joint, the skeleton joints were extracted directly from the Kinect, which generates the gait feature. On the other hand, H-joint distance is a feature of distance between the hip joint with other skeleton joints. Prior to the gait feature extraction, the skeleton joints provided by Kinect were pre-processed in order to standardize the size of the skeleton image as well as to detect the gait feature within a full gait cycle. To classify gait patterns according to its own group, a multi-layer perceptron was employed in the pattern recognition stage. Results show that a perfect recognition of human gait $(100 \%)$ was attained for the frontal view using the feature of H-joint distance at the optimal multi-layer perceptron (20 hidden units).
\end{abstract}

Keywords: human gait recognition; Kinect; oblique view; frontal view; gait cycle.

Author Correspondence, e-mail: nooritawati@ieee.org

doi: http://dx.doi.org/10.4314/jfas.v9i4s.1

\section{INTRODUCTION}

Recently, human gait has been studied extensively as a biometric feature in the human recognition system. Compared to other biometric features, gait offers many advantages in 
human recognition such as being able to identify a person in low resolution images with no requirement for closed-interaction between the person and equipment and requirement of simple image acquisition equipment [1-4].

A gait analysis begins by recording human gait using image acquisition devices such as video camera. The gait features for each video frame can be extracted using model-based approach or model-free approach before being fed into the pattern classifier. The model-based approach is an approach that recognizes human gait based on human body parts such as the stick-figure model [5]. The stick-figure model generates skeleton joints from the silhouette of detected body. In the model-free approach however, human gait is detected based on the silhouette shapes of the walking human. Compared to the model-free approach, the model-based approach suffers from complex algorithms and poor recognition performance due to its difficult process in segmenting human body from the background as well as inaccurate model fitting in generating skeleton joint points. However, this approach has been proven to give good performance by employing static and dynamic features that are generated from the skeleton joints [6-7]. Furthermore, this approach is robust to external factors such as footwear, clothing and carrying goods [8].

Recent researches attempt to overcome the drawback of the model-based approach by applying a new generation of image acquisition devices such as Vicon and Kinect in the model-based human gait recognition system [9-12]. Compared to the standard video camera, Vicon and Kinect are able to generate images along their gait features without involving complex processes. Nevertheless, due to expensive cost and tedious process which requires marker-based devices, Vicon is less preferred in the proposed study.

Kinect is an inexpensive device used primarily for the xbox gaming. The Kinect has been proven able to remove background artifact of the images and automatically generate information of 20 skeleton joint locations in 3D space. The skeleton joints were derived from the depth image of the captured body silhouette [7]. The depth image is generated by a projection of infrared light from the IR emitter to a human in the range of $0.8 \mathrm{~m}$ to $4 \mathrm{~m}$ from the Kinect. Due to its good performance such as robust, effective, reliable, high speed measurement and accessible, application of the Kinect has been extended to other areas 
especially in biometric area [13-14].

Based on the current review, the application of the Kinect sensor in the human recognition technique has begun in 2012. An indoor environment and normal gait speed were common control parameters used in the data acquisition stage [6-7, 11-12, 15-16]. The recognition of human gait using Kinect was mostly performed in lateral view [6-7, 11-12], which signifies insufficient study on the recognition of human gait in the oblique and frontal view.

This study proposed the recognition of human gait in oblique and frontal views due to insufficient analysis and research on this matter. New static gait features derived from the skeleton joints were extracted and analyzed in order to attain potential gait feature for the recognition of human gait in the oblique and frontal views. Multi-layer perceptron (MLP) classified the static gait feature patterns according to their groups. The recognition accuracy was computed to gauge the recognition performance and optimization on the MLP. This was examined throughout the study.

\section{RELATED WORKS}

The performance of the model-based approach using the Kinect sensor was primarily studied in lateral view [17]. The human was asked to walk perpendicularly facing the Kinect. An analysis on potential gait feature to represent the uniqueness of human was the most frequent task performed $[7,[11-12,17]$. The gait feature can either be classified as static feature or dynamic feature. Static feature can be defined as a feature that barely changes throughout the walking sequence, for instance, the length of arms and height. While feature that changes according to time frame is categorized as dynamic feature.

In 2012, in [17] static and dynamic gait features of nine subjects based on the skeleton points provided by Kinect was analyzed. To validate static gait feature, gait feature of subjects in idle position was compared to gait feature of lateral walking subjects with both facing the Kinect. From the 20 skeleton joints, 13 sets of features were extracted and classified by using naïve Bayes, One Rule algorithm (1R) and C4.5 algorithm. The highest recognition accuracy, 91\% was obtained when using naïve Bayes on the combination of static features; height, length of leg, length of torso and length of the upper left arm. 
In 2013, in [12] gait features in a half gait cycle was analyzed. The researchers recognized 10 subjects in lateral view from the Kinect. An automatic half gait cycle detection was introduced in this study. The detection was performed based on the distance between the left ankle and right ankle in the $\mathrm{x}$-coordinate. To construct the feature pattern, the mean of the gait features for each half gait cycle was computed. Two sets of feature namely the static feature and hybrid feature were proposed in this study. The combination of area of upper body part and lower body part was computed for the static feature. The hybrid feature comprises of areas below the hip and the distances between the body centroid and centroid that derived from the upper and lower limb joints. MLP has been used in the feature selection as well as the pattern classification stage. The study has shown that the set of static feature was the most significant feature compared to the hybrid, which obtained $68 \%$ of correct classification. The best performance is matched with the content of the feature set since the feature is derived based on the spreading of hand and leg; which is a unique gait for an individual. This study would has been more interesting if the researchers had classified all gait features by normalizing frame number for each half gait cycle rather than maintaining the averageness of the features. Furthermore, the highest recognition accuracy of $62 \%$ was obtained when using the hybrid features with the feature selection method.

An investigation on gait features sets within full gait cycle was performed by [11]. They developed various sets of gait features from skeleton joints that were provided by the Kinect for lateral view. Seven different sets of feature which consist of static and dynamic features have been generated from 15 subjects. An automatic gait cycle algorithm was proposed. They calculated ankle distance in xyz-coordinates and considered three consecutives of local minima as one full gait cycle. Statistical analysis such as mean and standard deviation has been performed on the gait features for each full gait cycle. For recognition, they employed multi-layer perceptron (MLP), support vector machine (SVM) and J48 algorithm. The selection of features using principal component analysis (PCA) was also employed in this study; however they performed only on two out of seven feature sets. As a result, the best recognition accuracy was obtained with a MLP classifier on features of length of step, distance between adjacent joint and angle. Furthermore, features of the lower body part 
attained higher accuracy compared to the upper body part. However, this result may involve potential measurement error as the researcher suspected that the placement of the Kinect lead to poor results due to most of the upper body parts being hidden. It was also determined that the worst classification accuracy was obtained when PCA was being employed on the feature sets.

In 2014, in [7] 10 subjects were classified through their gait in lateral view. They generated two datasets from static and dynamic features within each full gait cycle. They performed dynamic time warping (DTW) on the gait signal in order to normalize time frames for training and test datasets. As a result, $82 \%$ of recognition accuracy was obtained when using the combination of static and dynamic features.

From the mentioned researches, it can be determined that there is no normalization of skeleton image for the recognition of human gait in the lateral view. The normalization of skeleton image was not required since the size of the skeleton image is similar for the whole time frames. However, problem arose for oblique view and frontal view. The size of skeleton image increases as the human approaches the Kinect. $[15,18]$. As such, there is need for an additional process known as pre-processing to normalize the skeleton images. Generally, the pre-processing stage could enhance the recognition performance in the pattern recognition stage [19]. However, to date, only [15, 20] have carried out the pre-processing stage in the recognition of human gait

In [15], the researchers proposed a method called trajectory processing in the pre-processing stage. The main purpose of the proposed method was to normalize the size of skeleton image of 20 subjects in frontal view. The size of all the skeletons in the $\mathrm{x}$-axis and $\mathrm{y}$-axis were scaled at 100 pixels of distance between the shoulder center and the hip center throughout the walking sequence. In addition, a relative movement of skeleton joints together with the rest was computed. In this computation, the researchers employed the hip joint as a reference joint. In the classification stage, a covariance analysis was performed on the different sets of feature and found that the combined features obtained higher recognition accuracy compared to the single feature. The highest recognition accuracy, $93.33 \%$ was achieved by using a combination of spine, right arm and left leg. 
On the other hand, the study in [20] performed the pre-processing stage in order to determine the direction of the walking subject. The researchers captured a video of 17 walking subjects in various angle views such as lateral, frontal and oblique using Kinect. DTW was employed on static and kinematic features and the nearest neighbor was used as the pattern classifier. As a result, 51\% of recognition accuracy was achieved when using both static and kinematic features [23].

Studies on the recognition of human gait in frontal view without the pre-processing stage was performed by $[18,16]$. Gait features were extracted directly from the skeleton joints provided by Kinect. In [18], sequences of human gait in spatial-temporal were transformed to spatial domain before being fed into the Content-based image retrieval (CBIR) classifier. Results have shown that further analysis should be done in order to verify the proposed method in the recognition of human gait. In [16], a low recognition performance was attained for the recognition of 20 subjects who walked in frontal view. An investigation into static and dynamic features was also performed in this study. For recognition, K-means unsupervised was employed. As a result, they only obtained $51 \%$ of recognition accuracy. Furthermore, the researchers determined that the dynamic features consisting of knees, elbows and head are the most significance features. Considering all of these evidences, it appears that:

- The Kinect has been proven as a reliable device in human gait recognition.

- There are limited analysis on potential gait features for oblique and frontal views.

- Majority of the researchers focus on human gait recognition in lateral view, which does not require pre-processing for skeleton normalization purpose.

\section{METHODOLOGY}

The recognition of human gait in oblique and frontal views was performed as shown in Fig. 1. The human is recognized after the sequence of the video frame passed through stage of data acquisition, pre-processing, feature extraction and lastly pattern recognition. This process is implemented using MATLAB r2015a. 


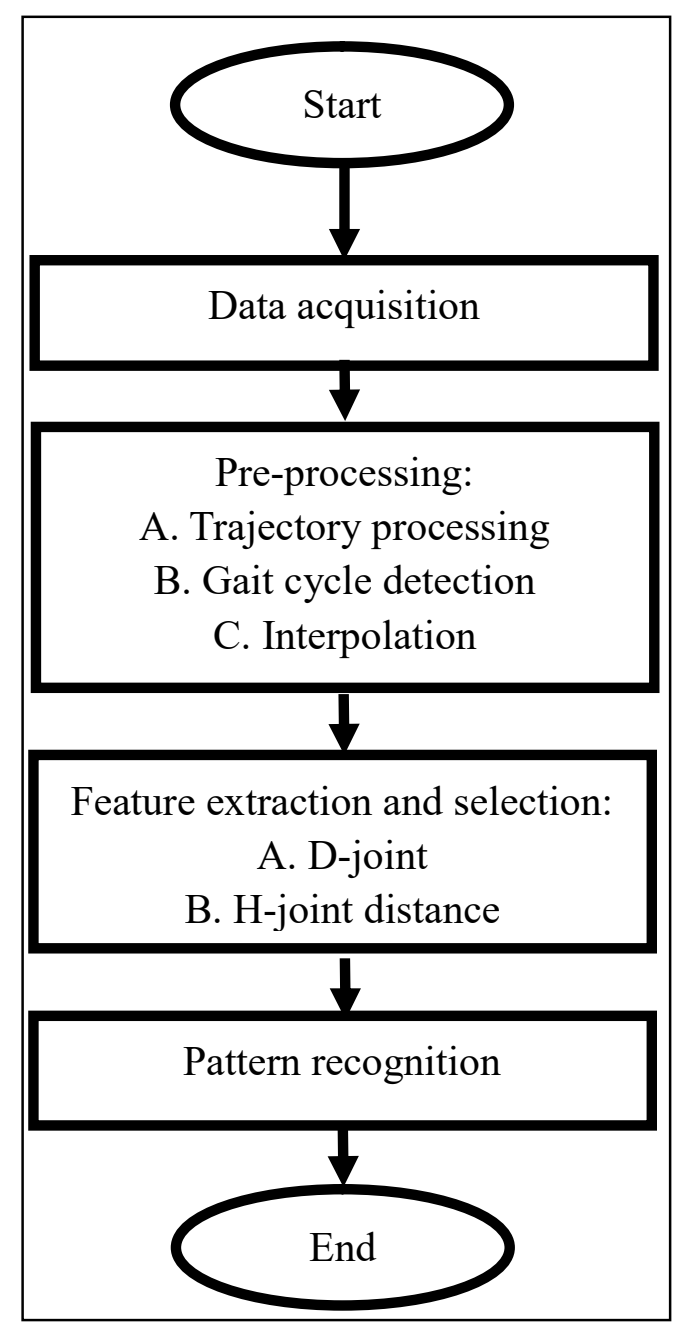

Fig.1. The proposed design

\subsection{Data Acquisition}

Data acquisition was implemented in an indoor environment and the outfit of the subjects were standardized to long pants and cover shoes. Fig. 2 shows the layout measurement for the data acquisition. The Kinect sensor recorded a video frame of the 12 walking subjects in RGB and depth image (RGB-D) along with their skeleton joint points. For oblique view, the subjects were asked to walk in a $45^{\circ}$ from the upper right side of the Kinect. As for the frontal view, the subjects were asked to walk towards the Kinect sensor for 10 times in their common gait with normal speed in both the frontal and oblique view. Thus, 20 walking sequences were generated for each subject. 


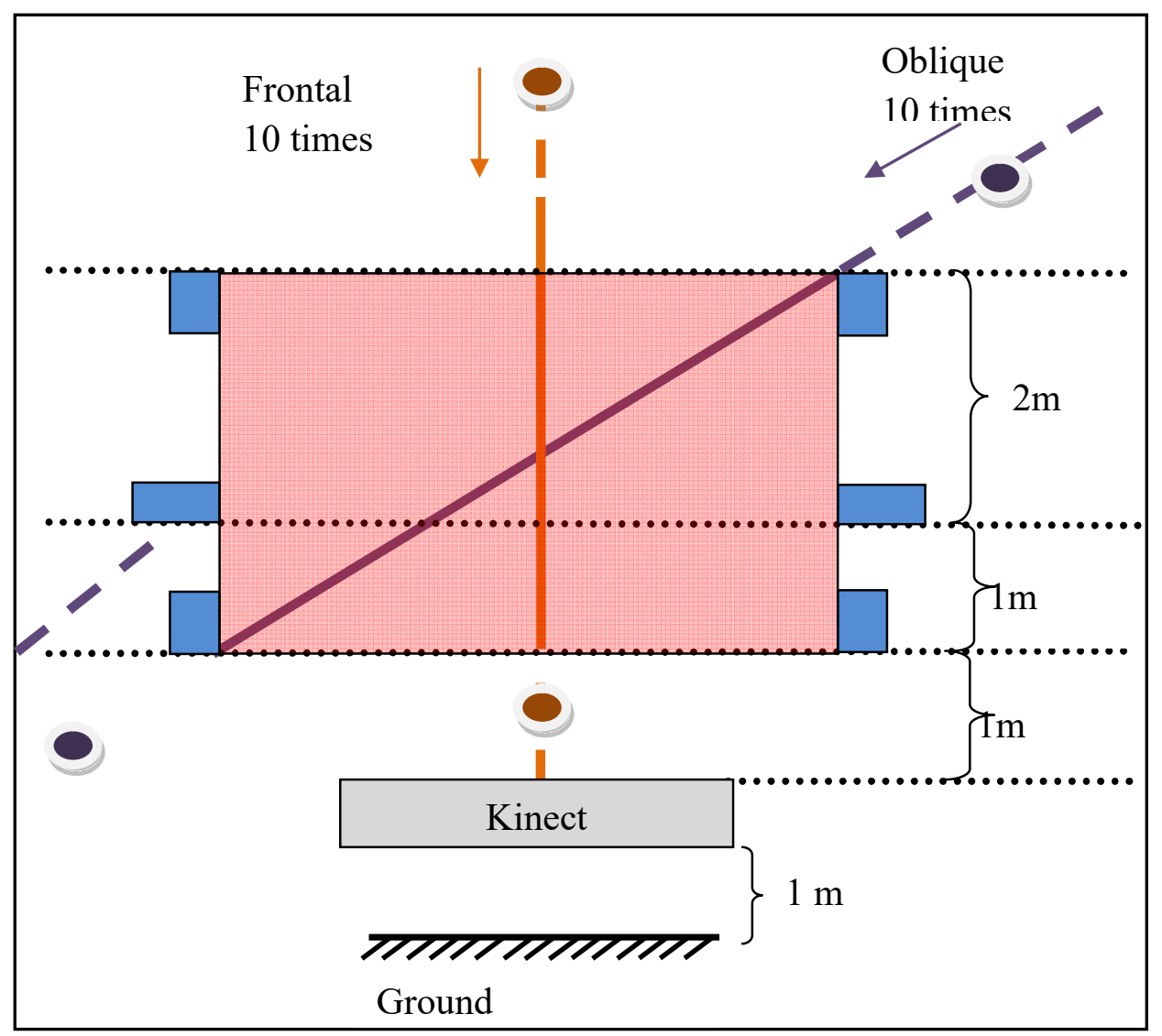

Fig.2. Layout for frontal and oblique view

\subsection{Pre-Processing}

There were three steps involved in the pre-processing stage; trajectory processing, gait cycle detection and interpolation. In the trajectory processing, skeleton image was normalized and the movement of skeleton joints respect to each other was computed. Then, detection of full gait cycle was carried out in order to extract gait feature within the full gait cycle. Finally, the extracted feature for each gait cycle was standardized at same size of features in the interpolation.

\subsubsection{Trajectory Processing}

The main aim of this stage is to normalize skeleton joint points. The normalization is employed in xyz-coordinates. Fig. 3 depicts the steps involved in the pre-processing stage. At first, the skeleton joint points obtained from the video frames were standardized at the reference height. In this study, the reference height refers to the height of the subject at a half of the total of the video frame. Then, the relative movement of the skeleton joints with respect to a constant head joint was computed. 


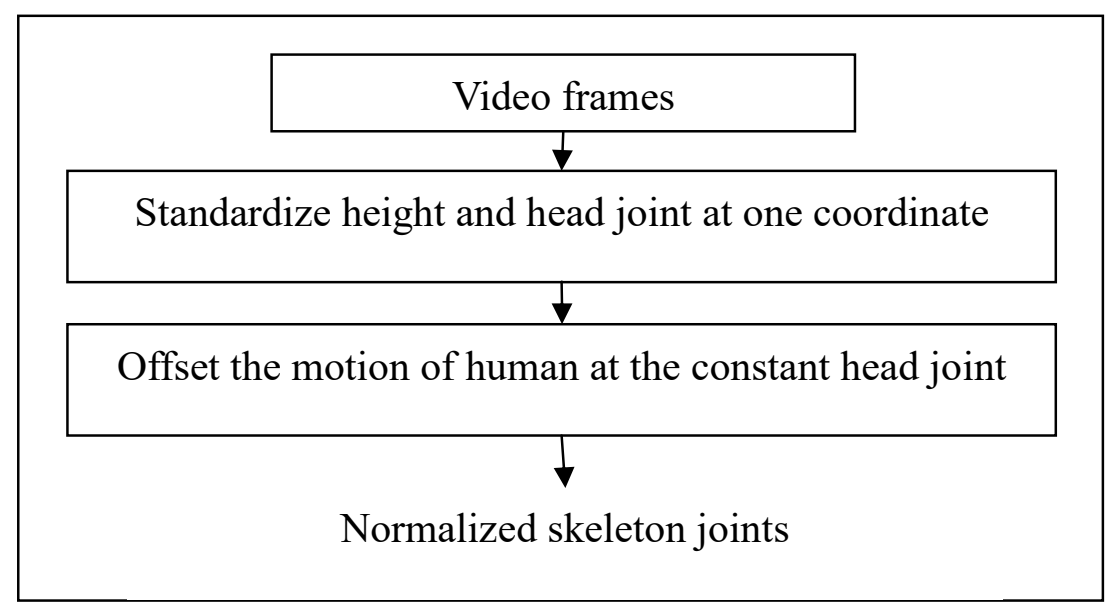

Fig.3. Steps in pre-processing stage

\subsubsection{Gait Cycle Detection}

In this study, features were generated within a gait cycle. Gait cycle is a period of a sequence of the same foot touching the ground twice. Generally, the gait cycle consists of different number of frames even though they are for the same subject. The various number of frames could cause inequality in the size of feature pattern, which can lead to difficulty in the classification stage. Earlier studies fixed a gait cycle into 32 frames, since that is the typical human gait cycle [21-22]. However, through observation in this study, the obtained gait cycle was less than 32 frames. Hence, the detection of gait cycle was computed based on distance vector between the right ankle and left ankle which was implemented by [11], except for the smoothing algorithm. The smoothing algorithm was changed to Savitzky-Golay's moving average algorithm, since it offers clearer gait signal during the analysis performed in this study. Local minima which is a transition from negative slope to positive slope is detected from the distance vector and one full gait cycle as three consecutive local minima. As a result, 1 to 3 gait cycles was obtained for oblique view and 1 to 2 gait cycles was obtained for frontal view. To avoid bias performance in the classification stage, one gait cycle has been selected for each walking sequence per subject. This resulted in 120 gait cycles for 12 subjects.

\subsubsection{Interpolation}

Various numbers of frame ranged from 8 frames to 25 frames were obtained for 120 gait cycles of frontal view, while 15 frames to 28 frames were obtained for oblique view. It causes unequal size of input features in the classification stage. Hence, an additional approach has to be added in order to synchronize the input feature size. Through the interpolation process, the number of frame for each gait cycle can be fixed at the same size. Interpolation is a mathematical algorithm commonly used for curve fitting. It is a process of generating new data points within a discrete set of an original data. As a consequence, the shape of the 
constructed curve is almost identical to the original curve. There are few methods in interpolation such as linear, polynomial and spline. In this study, spline interpolation has been chosen since it employs low-degree polynomials in finding new data points and smoothing the set of data point as well. Hence, throughout the interpolation for frontal view, the frame number of gait cycle was fixed at the maximum frame which is 25 frames. As a result, the dimension of gait signal for one gait cycle is [60 x 25]. For standardizing purpose, the same frame number of frontal view was selected for oblique view.

\subsection{Feature Extraction}

This study mainly focuses in designing new gait features namely D-joint feature and H-joint distance feature. An extensive analysis on potential gait feature for body part was carried out as well. The extracted feature can be divided further into different parts of the body.

\subsubsection{D-Joint Feature}

This feature set is constructed from the skeleton joints that were directly extracted from the Kinect. It can be divided into different parts of the body:

- Feature 1: All skeleton joints of a body are combined for this set of feature.

- Feature 2: This set of feature is designated for the upper body part only. It contains the joint of spine, center shoulder, head, left shoulder, left elbow, left wrist, left hand, right shoulder, right elbow, right wrist and right hand.

- Feature 3: Joints for lower body part such as spine, hip, left foot, right hip, right knee, right ankle, right foot, left hip, left knee and left ankle are constructed for this feature set.

- Feature 4: This set consists of joints from the right side of body, including the head, center shoulder, spine, center hip, right shoulder, right elbow, right wrist, right hand, right hip, right knee, right ankle and right foot.

- Feature 5: This set consists of joints from the left side of the body, including the head; center shoulder, spine, center hip, left shoulder, left elbow, left wrist, left hand, left hip, left knee, left ankle and left foot.

\subsubsection{H-Joint Distance}

This feature set is constructed from the computation of distance between the hip center with other skeleton joints, as listed in Table 1 and Table 2. The hip center was chosen as a reference point since it has the most consistent joint point compared to other joints. The distance between the joints are calculated for each frame of the gait cycle using Euclidean distance:

$d_{i}=\sqrt{\left(h i p_{-} c_{x}-j_{i x}\right)^{2}+\left(h i p_{-} c_{y}-j_{i y}\right)^{2}+\left(h i p_{-} c_{z}-j_{i z}\right)^{2}}$ 
where hip_c is the hip center and $\mathrm{j}$ is the other joint.

Table 1. Skeleton joints for upper and lower parts of body

\begin{tabular}{cc}
\hline Body Part & Name of Skeleton Joint \\
\hline Upper part of body & Left and right shoulder \\
Left and right elbow \\
Left and right wrist \\
Left and right hand \\
Left and right hip \\
Left and right knee \\
Left and right ankle \\
Left and right foot
\end{tabular}

Table 2. Skeleton joints for left and right parts of body

\begin{tabular}{ccc}
\hline Body Part & \multicolumn{2}{c}{ Name of Skeleton Joint } \\
\hline Left side of body & Left shoulder & Left hip \\
& Left elbow & Left knee \\
Left wrist & Left ankle \\
& Left hand & Left foot \\
Right side of body & Right shoulder & Right hip \\
& Right elbow & Right knee \\
& Right wrist & Right ankle \\
& Right hand & Right foot
\end{tabular}

- Feature 6: Distance between hip center joint and each skeleton joints as listed in Table 1 and Table 2 is designated for this set of feature.

- Feature 7: For this set of feature, distance between joint of hip center and upper part of the body were selected.

- Feature 8: This set of feature is constructed for the lower part of the body. The distance between the hip center and the lower part of the body is combined in this set.

- Feature 9: This set consists of distance between the joint of the hip center and the skeleton joints at the right side of the body.

- Feature 10: This set consists of distance between the joint of hip center and the skeleton joints at the left side of the body. 
Table 3 shows the distribution of feature number for the D-joint and $\mathrm{H}$-joint distance feature. As shown below, a large number of feature belong to Feature 1 as skeleton joints for the whole body was extracted.

Table 3. Distribution of feature number for D-joint and H-joint distance feature

\begin{tabular}{ccc}
\hline Feature Set & Description & Number of Features \\
\hline Feature 1 & D-joint for the whole body & 1500 \\
Feature 2 & D-joint for upper part body & 825 \\
Feature 3 & D-joint for lower part body & 750 \\
Feature 4 & D-joint for right side body & 900 \\
Feature 5 & D-joint for left side body & 900 \\
Feature 6 & H-joint distance for the whole body & 400 \\
Feature 7 & H-joint distance for upper part body & 200 \\
Feature 8 & H-joint distance for lower part body & 200 \\
Feature 9 & H-joint distance for right side body & 200 \\
Feature 10 & H-joint distance for left side body & 200 \\
\hline
\end{tabular}

\subsection{Pattern Recognition}

In this stage, the various sets of feature were classified according to the subject's information. This process is compares the extracted features with features that have been obtained by the MLP classifier. Hidden unit varied from 5 to 100 with an increment of 5 in order to obtain the optimum classifier model. Before the input feature was fed to MLP, it was normalized and scaled in the range -1 to +1 . The input feature was divided randomly at the ratio $70: 15: 15$ of training, validation and testing. The experiment was repeated for 10 times and the recognition performance was evaluated based on the average recognition accuracy. Recognition accuracy is the percentage of correct recognition to total of sample.

\section{RESULTS AND DISCUSSION}

\subsection{Pre-Processing}

\subsubsection{Trajectory Processing}

To validate the proposed method of trajectory processing, observation on skeleton image and gait signal and correlation analysis was conducted. Fig. 4 shows the observation of skeleton image from the frontal view at different time frame. It can be seen that the size of the skeleton image is increases as the subject approaches the Kinect. After trajectory processing, the 
skeleton size has been normalized to the reference height as shown in Fig.4. It can also be seen that the coordinate of the walking subject was fixed at one spot.

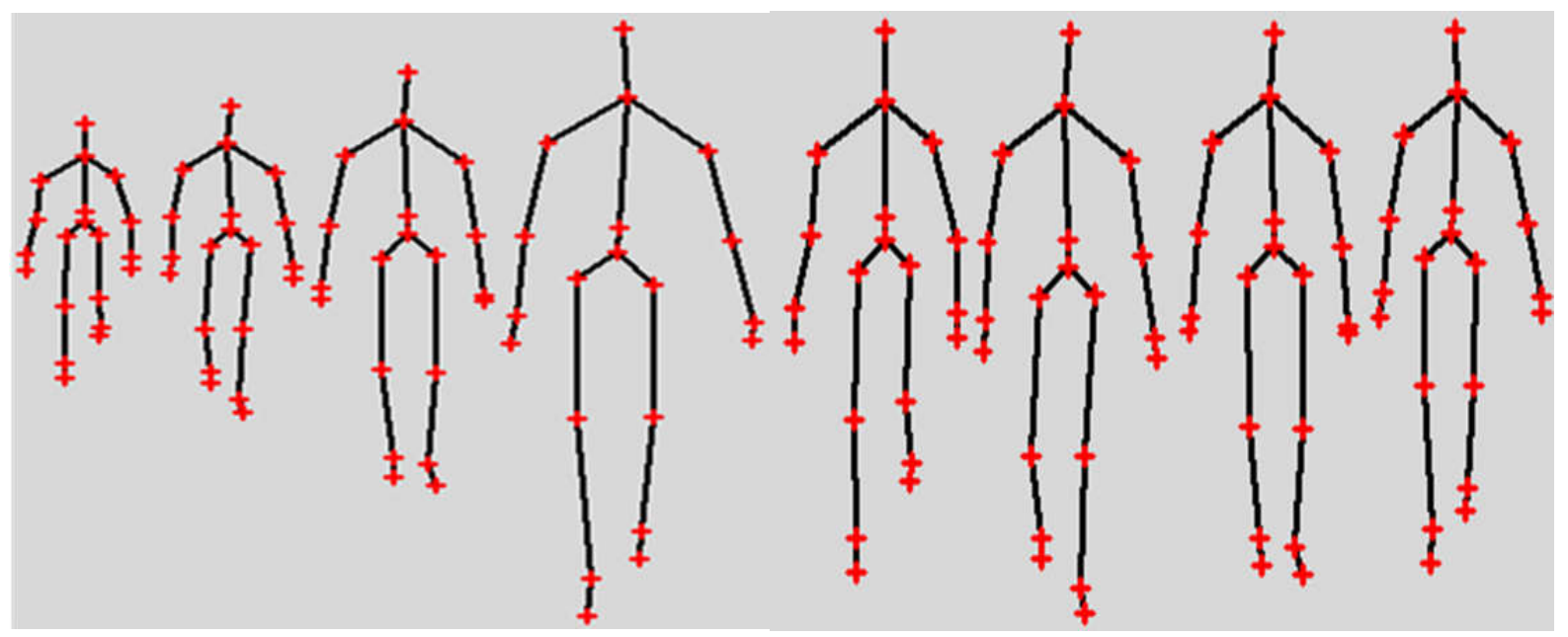

(a)

(b)

Fig.4. Skeleton of walking subject at different time frame: (a) before normalization process (b) after normalization process

Fig. 5 shows the signal of the hip center joint for one walking sequence before and after normalization for the oblique view. As shown below, the normalized signal maintained the same pattern as the original signal.

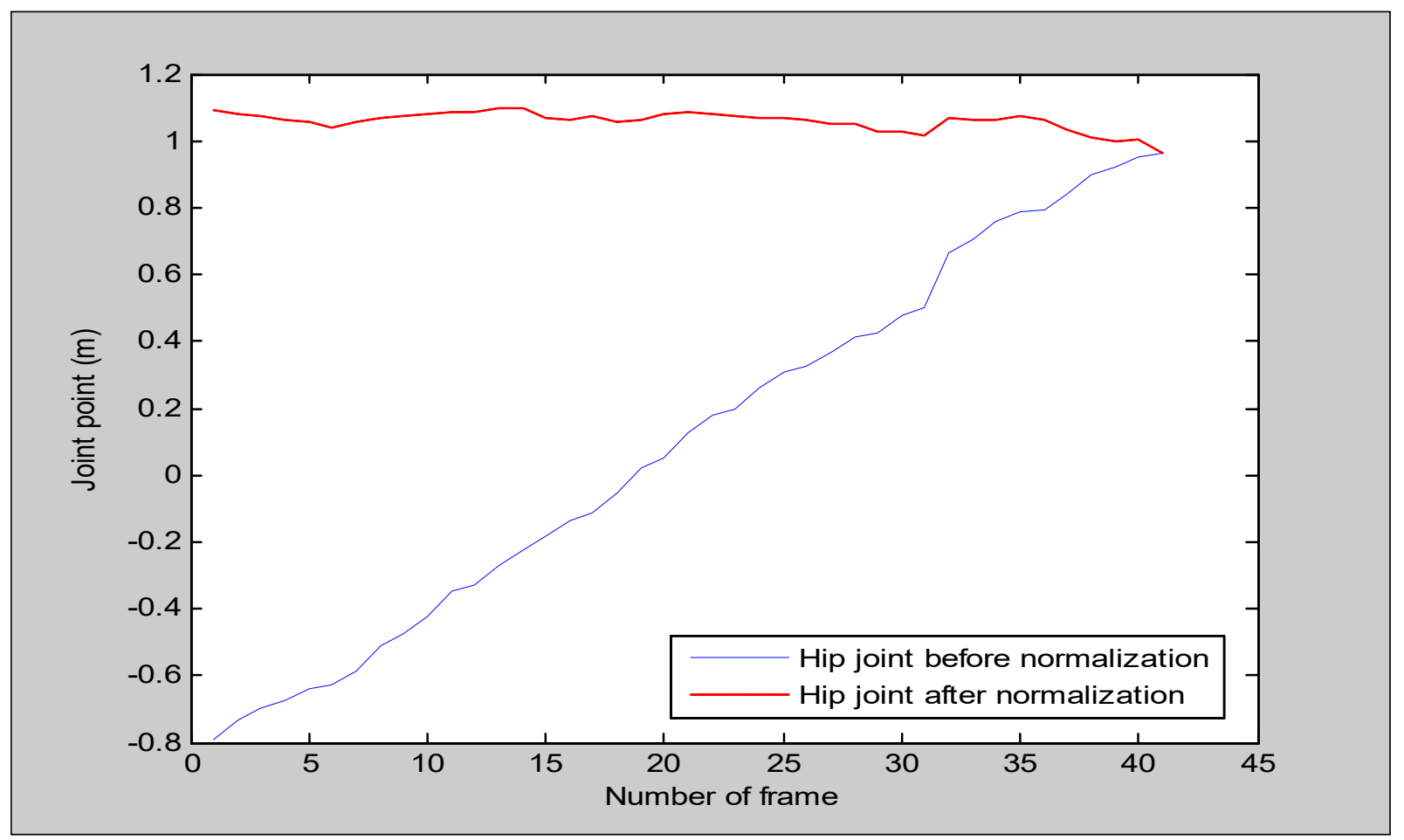

Fig.5. Signal of hip center joint before and after normalization in x-coordinate

A correlation analysis was computed to verify the similarity between the original signal and the normalized signal. Results show that the correlation for both the oblique and frontal views 
were in the range of 0.8 to 1 . Score of 1 indicates that the original signal and the normalized signal have a perfect similarity.

\subsubsection{Detection of Gait Cycle}

Comparisons between automatic and manual gait cycle detection was performed in order to evaluate the performance of the automatic detection. Based on the observation, the accuracy of correct detected gait cycle, $91.43 \%$ is attained for the oblique view and $99.30 \%$ for the frontal view.

\subsection{Pattern Classification}

Table 4 and Table 5 show the recognition performance of human gait for D-joint feature and $\mathrm{H}$-joint distance feature, respectively. The highest recognition accuracy, $100 \%$ has been obtained at 20 hidden units when using the feature of H-joint distance for the frontal view. The lowest accuracy is obtained at the lower part of the body for all categories of features for both the oblique and frontal views. This may happen due to the inaccuracy of the joint location at the lower part of the body. It was also an evident that a large hidden unit is required in recognizing human gait using skeleton joints for the oblique view compared to the frontal view.

\subsubsection{Oblique}

Occlusion occurs at the right side of body as most of the skeleton joints have been hidden from the Kinect. Supposedly, the lowest recognition accuracy will be obtained from the right side as opposed to the left side. However, a higher accuracy of $89.44 \%$ was obtained at the right side when using features that generate directly from the skeleton joints (D-joint). In contrast to the D-joint, an acceptable recognition accuracy was obtained when the gait recognition was performed using joint distance features (H-joint distance). The recognition accuracy of the left side body is higher than that of the right side body. It seems that the gait recognition using feature generated from the H-joint distance can be performed accurately. Furthermore, the highest recognition accuracy (90.00\%) was attained for features of the whole body.

\subsubsection{Frontal}

For frontal view, the highest recognition of human gait using the $\mathrm{H}$-joint distance achieved $100 \%$ accuracy compared to D-joint (94.44\%). It can be observed that the same accuracy was attained for the whole body part and the left side of the body for both the D-joint feature and H-joint distance feature. The $100 \%$ correct recognition is matched with the application of Kinect since it is designated for the detection of human in the frontal view. 
Table 4. Recognition performance for D-joint feature

\begin{tabular}{|cccccc} 
& & \multicolumn{2}{c}{ Oblique } & \multicolumn{2}{c}{ Frontal } \\
\cline { 3 - 5 } & Feature Set & $\begin{array}{c}\text { Hidden } \\
\text { Number }\end{array}$ & $\begin{array}{c}\text { Recognition } \\
\text { Accuracy }\end{array}$ & $\begin{array}{c}\text { Hidden } \\
\text { Number }\end{array}$ & $\begin{array}{c}\text { Recognition } \\
\text { Accuracy }\end{array}$ \\
\cline { 3 - 6 } 1 & All body & 70 & 80 & 25 & 90 \\
\hline 2 & Upper body part & 125 & 88.33 & 35 & $\mathbf{9 4 . 4 4}$ \\
3 & Lower body part & 120 & 64.44 & 50 & 71.11 \\
4 & Right side body & 170 & $\mathbf{8 9 . 4 4}$ & 25 & 83.33 \\
5 & Left side body & 175 & 74.44 & 25 & 90
\end{tabular}

Table 5. Recognition performance for $\mathrm{H}$-joint distance feature

\begin{tabular}{|llcccc} 
& & \multicolumn{2}{c}{ Oblique } & \multicolumn{2}{c}{ Frontal } \\
& Feature Set & Hidden & Recognition & Hidden & Recognition \\
& Number & Accuracy & Number & Accuracy \\
\hline 6 & All body & 35 & $\mathbf{9 0}$ & 35 & $\mathbf{1 0 0}$ \\
7 & Upper body part & 40 & 78.89 & 45 & 95.56 \\
8 & Lower body part & 25 & 75.56 & 30 & 86.67 \\
9 & Right side body & 80 & 83.33 & 95 & 92.78 \\
10 & Left side body & 40 & 88.33 & 20 & $\mathbf{1 0 0}$
\end{tabular}

\section{CONCLUSION}

This project proposed new gait features, namely D-joint and H-joint distance features for the recognition of human gait in the oblique and frontal views. This study has shown that the feature of $\mathrm{H}$-joint distance proves to be the most significant feature for both views. Moreover, it can be observed that among all the features of the oblique and frontal views, features at the lower part of the body were less significant due to the lowest recognition accuracy. The most significant feature could be obtained from the whole body and upper body part. Further studies on gait features in oblique and frontal view with large samples of subjects need to be carried out in order to operate the recognition technique in real world applications.

\section{ACKNOWLEDGEMENTS}

This research is funded by Ministry of Higher Education (MOHE) Malaysia under the Niche Research Grant Scheme (NRGS) Project No: 600-RMI/NRGS 5/3 (8/2013) and Research Grant Scheme Project No: 600-RMI/DANA5/3/PSI (195/2013) for supporting this journal 
publication. The authors wished to thank Human Motion Gait Analysis (HMGA) Laboratory, IRMI Premier Laboratory, Research Management and Innovation (IRMI), Universiti Teknologi MARA (UiTM), Malaysia for the instrumentation and experimental facilities provided as well as Faculty of Electrical Engineering UiTM Shah Alam for all the support given during this research.

\section{REFERENCES}

[1] Ariyanto G, Nixon M S. Model-based 3D gait biometrics. In IEEE International Joint Conference on Biometrics, 2011, pp. 1-7

[2] Iwashita Y, Uchino K, Kurazume R. Gait-based person identification robust to changes in appearance. Sensors, 2013, 13(6):7884-7901

[3] Cattin P C. Biometric authentication system using human gait. Phd thesis, Switzerland: Swiss Federal Institute of Technology ETH Zürich, 2002

[4] Gafurov D. A survey of biometric gait recognition: Approaches, security and challenges. In Annual Norwegian Computer Science Conference, 2007, pp. 19-21

[5] Wheat J, Fleming R, Burton M, Penders J, Choppin S, Heller B. Establishing the accuracy and feasibility of Microsoft Kinect in various multi-disciplinary contexts. EFL Kinect Project Report, England: Sheffield Hallam University, 2012

[6] Jianwattanapaisarn N, Cheewakidakarn A, Khamsemanan N, Nattee C. Human identification using skeletal gait and silhouette data extracted by Microsoft Kinect. In IEEE 15th International Symposium on Soft Computing and Intelligent Systems Joint 7th International Conference on and Advanced Intelligent Systems, 2014, pp. 410-414

[7] Jiang S, Wang Y, Zhang Y, Sun J. Real time gait recognition system based on Kinect skeleton feature. In Asian Conference on Computer Vision, 2014, pp. 46-57

[8] Sivapalan S. Human identification from video using advanced gait recognition techniques Phd thesis, Brisbane: Queensland University of Technology, 2014

[9] Stone E, Skubic M. Evaluation of an inexpensive depth camera for in-home gait assessment. Journal of Ambient Intelligence and Smart Environments, 2011, 3(4):349-361 [10]Granata C, Salini J, Ady R, Bidaud P. Human whole body motion characterization from embedded Kinect. In IEEE 4th International Conference on Cognitive Infocommunications, 
2013, pp. 133-138

[11] Dikovski B, Madjarov G, Gjorgjevikj D. Evaluation of different feature sets for gait recognition using skeletal data from Kinect. In 37th IEEE International Convention on Information and Communication Technology, Electronics and Microelectronics, 2014, pp. 1304-1308

[12] Sinha A, Chakravarty K, Bhowmick B. Person identification using skeleton information from Kinect. In International Conference on Advances in Computer-Human Interactions, 2013, pp. 101-108

[13] Munsell B C, Temlyakov A, Qu C, Wang S. Person identification using full-body motion and anthropometric biometrics from Kinect videos. In European Conference on Computer Vision, 2012, pp. 91-100

[14] Procházka A, Vyšata O, Vališ M, Yadollahi M. The MS Kinect use for 3d modelling and gait analysis in the MATLAB environment. In P. Byron (Ed.), Technical computing Prague 2013. Prague: The Institute of Chemical Technology, 2013, pp. 270-275

[15] Kumar M S, Babu R V. Human gait recognition using depth camera: A covariance based approach. In 8th ACM Indian Conference on Computer Vision, Graphics and Image Processing, 2012, pp. 1-6

[16] Gianaria E, Balossino N, Grangetto M, Lucenteforte M. Gait characterization using dynamic skeleton acquisition. In IEEE 15th International Workshop on Multimedia Signal Processing, 2013, pp. 440-445

[17] Preis J, Kessel M, Werner M, Linnhoff-Popien C. Gait recognition with Kinect. In 1st International Workshop on Kinect in Pervasive Computing, 2012, pp. P1-P4

[18] Milovanović M, Minović M, Starcević D. New gait recognition method using Kinect stick figure and CBIR. In 20th IEEE Telecommunications Forum, 2012, pp. 1323-1326

[19] Alginahi Y. Chapter 1-Preprocessing techniques in character recognition. In M. Mori (Ed.), Character recognition. Rijeka: InTechOpen, 2010, pp. 1-20

[20] Cheewakidakarn A, Khamsemanan N, Nattee C. View independent human identification by gait analysis using skeletal data and dynamic time warping. In 14th International Symposium on Advanced Intelligent Systems, 2013, pp. 1-6 
[21] Xiao F, Hua P, Jin L, Bin Z. Human gait recognition based on skeletons. In IEEE International Conference on Educational and Information Technology, 2010, pp. 83-87

[22] Zhang R, Vogler C, Metaxas D. Human gait recognition. In IEEE Conference on Computer Vision and Pattern Recognition Workshop, 2004, pp. 1-8

[23] Hasan C Z, Jailani R, Tahir N M, Yassin I M, Rizman Z I. Automated classification of autism spectrum disorders gait patterns using discriminant analysis based on kinematic and kinetic gait features. Journal of Applied Environmental and Biological Sciences, 2017, $7(1): 150-156$

\section{How to cite this article:}

Sahak R, Yassin I, Tahir NM, Zaman FHK, Zabidi A.Recognition of human gait in oblique and frontal views using kinect. J. Fundam. Appl. Sci., 2017, 9(4S), 1-18. 